\title{
Search of critically important combinations of objects of the gas industry from the positions of the system operability
}

\author{
Sergey Vorobev $^{1 *}$, Sergey Senderov ${ }^{1}$, Alexey Edelev ${ }^{1}$ \\ ${ }^{1}$ Melentiev Energy Systems Institute, 130 Lermontov str., Irkutsk, Russia
}

\begin{abstract}
The paper is devoted to the search for critical combinations of gas industry objects from the standpoint of system operability. Critically important is the object partial or complete failure which can cause significant damage to the country from the fuel and energy complex in general or within the framework of a separate energy system. It is possible to failure of a critical and other object of the system. The paper presents the main major critical combinations of gas industry objects. Their significance and impact on the efficiency of the gas industry in Russia is shown. Conclusions are made about the expediency of searching for critical combinations of gas industry objects from the standpoint of system operability.
\end{abstract}

\section{Introduction}

One of the main aspects of ensuring energy security is the creation of conditions for the greatest possible degree of satisfaction of consumers with energy resources in emergency situations [1]. The study of this aspect of energy security requires the identification of critical objects for the fuel and energy complex in general and for individual energy systems. Critically important from the standpoint of energy security, it is necessary to consider an object whose partial or complete failure can cause significant damage to the country from the fuel and energy complex due to shortages to consumers of the necessary fuel and energy resources.

The increase in the number of major accidents in energy systems in recent years is due to the significant wear and tear of fixed assets, and the lack of significant financial investment in their reconstruction. Large-scale accidents in energy systems caused by the failure of various critical objects entail significant, sometimes irreparable, damage to consumers in the form of large short-term deliveries of final types of energy.

Current calculations show that in case of accidents at the major intersections of main gas pipelines, consumers of final types of energy resources in the regions will suffer significantly because of their deficiencies. In some cases, such deficits can amount to $30-50 \%$ of their need for certain periods of the year that are tense for consumption of energy resources. Along with the intersections of the main gas pipelines, potentially largescale nodal compressor stations, as well as linear sections of separate main gas transmission corridors, may be potentially dangerous for the operation of the system. From all this list of potentially dangerous facilities for the functioning of the system, the most significant, that is, critically important objects are currently being identified, followed by the development of measures aimed at improving the reliability of these objects to maintain uninterrupted fuel and energy supply to consumers.

Previously, a number of studies were conducted on the identification of critical objects in the gas transportation network. The list of crossings of main gas pipelines in the Unified Gas Supply System of Russia has been determined, the disruption of which will result in a relative shortage of daily gas supplies for the system as a whole of $5 \%$ or more $[2,3]$. Investigations were carried out to find and identify combinations of separate sections of main gas pipelines, the simultaneous disruption of which may lead to a significant shortage of daily gas supplies through the system $(5 \%$ or more) $[4$, 5].

At this stage of research of critical gas industry objects, critical combinations of gas industry objects are considered, that is, such facilities are identified whose failure, together with one of the critical objects, will lead to a much larger gas deficit in consumers than if they fail these objects separately. After identifying possible critical combinations of objects in each specific case, the possibility of bypassing bottlenecks is analyzed by shortterm increase in the capacity of individual sections of main gas pipelines. This is done with a view to minimizing gas shortages among consumers. As a result of such measures, the significance of a number of potential critical combinations of objects can be reduced. Thus, all possible critical combinations of gas industry objects will be identified and their list, ranked according to the degree of significance, will be formed. 


\section{Determination of critical elements in energy systems}

In the ongoing study, both for determining the critical objects themselves and for searching for critical combinations of objects, a flow model was used, which is the core of the software complex "Oil and Gas of Russia" [6, 7]. Its application makes it possible to determine the degree of satisfaction of gas needs within the country and to ensure export supplies. In addition, the "Oil and Gas of Russia" allows identifying "bottlenecks" - sections of the gas transmission network, which in some cases limit the production capacity of the system.

The flow distribution model in the Unified Gas Supply System of Russia in the "Oil and Gas of Russia" is designed to assess the production capabilities of the Unified Gas Supply System of Russia in conditions of various kinds of disturbances. The purpose of such studies is to minimize gas deficits at the consumption sites. The Unified Gas Supply System of Russia in the model is represented as a set of three subsystems: gas sources, main gas transport network and consumers.

When solving the problem of estimating the state of a system after a perturbation, the criterion of the optimality of the distribution of flows is the minimum gas deficit in the consumer with minimum costs for delivering gas to consumers. This problem can be solved by finding the maximum flow over the network. The problem of the maximal flow was investigated in [8] and is formulated as follows:

$$
f \rightarrow \max ,
$$

with follow conditions

$$
\begin{aligned}
& \sum_{i \in N_{j}^{+}} x_{i j}-\sum_{i \in N_{j}^{-}} x_{j i}=\left\{\begin{array}{c}
-f, j=O \\
0, j \neq O, S \\
f, j=S
\end{array}\right. \\
& 0 \leq x_{i j} \leq d_{i j}, \text { for all of }(\mathrm{i}, \mathrm{j}) .
\end{aligned}
$$

In this formulation, $f$ is the maximized variable corresponding to the maximum flow.

With complex schemes, which are the Russian and European gas transmission networks, there may be several solutions, that is, several possible maximum flows. Then it is advisable to talk about minimizing the cost of delivering gas to consumers and using the Basaker-Gowen algorithm [8]:

$$
\begin{aligned}
& \sum_{(i, j)} C_{i j} x_{i j} \rightarrow \min , \\
& x \in X^{*} .
\end{aligned}
$$

Its application allows us to determine the maximum flow of the resource at its minimum cost or the optimal volumes of daily gas from the underground gas storages, which maximally provide the given volumes of gas supply to consumers at the minimum costs for extraction, transportation of gas and its withdrawal from underground gas storages.

$O$ - the node number of the common source; $S$ - is the number of the common drain node; $N_{j}^{+}$- is a subset of edges entering into the node $j ; N_{j}$ - is the subset of outgoing edges from the node $j ; f-$ is the value of the total flow over the network; $x_{i j}$ - is the flow along the edge $(i, j) ; d_{i j}$ - restrictions on the flow along the edge $(i$, $j) ; X^{*}$ - is the set of solutions of problem (4)-(6); $C_{i j}-$ specific costs for gas transportation.

The node $O$ is connected by fictitious edges with all real sources of energy resources, and node $S$ - with all consumers.

In the flow distribution model, the Basaker-Gowen algorithm is used to calculate the maximum flow of minimum cost, which as a result allows us to determine the possible level of satisfaction of consumers with gas. As a result of implementation of various contingencies, there may be a gas shortage in consumers caused by a lack of capacity of certain sections of the gas pipelines. Bypassing such narrow or limiting the production capacity of the system's sites, in permissible amounts, will reduce the gas shortage that has arisen in the situation in question among consumers.

If there is a gas shortage in consumers caused by a shortage of capacity of the gas pipelines, other branches of the main gas pipelines, which are not affected by the violation in question, assume increased volumes of gas. In such a situation, the network load is changing and there may be a lack of capacity on other sections of the main gas pipelines.

The subsequent expansion of bottlenecks in the gas transportation network will allow to minimize gas shortages among consumers and makes an assessment and definition of possible critical combinations of gas industry objects as much as possible.

"Oil and Gas of Russia" has graphic capabilities, which allow the researcher to identify many potential bottlenecks in the analysis of calculation results, that is, objects that do not have a reserve of production capabilities. Most often, such facilities are either, or may become, in the future the reason for the shortage of the necessary amount of gas to consumers.

Even with the use of graphic capabilities of "Oil and Gas of Russia" it is impossible without additional modelling to find real bottlenecks, which at the moment really limit the ability of the gas transportation network to supply gas to consumers.

The result of solving the problem (1) - (5) is the value of the maximum flow in the system and the gas deficit in consumers. It is clear that in order to compensate for the gas deficit in consumers, the amount of flow should become more than calculated. Then the problem arises of increasing the carrying capacity of edges, in order to obtain a flow of the required value.

This problem of optimal network transformation [8] can be written in the following form:

with follow conditions

$$
f \rightarrow \max ,
$$

$$
\begin{gathered}
\sum_{i \in N_{j}^{+}}\left(x_{i j}+y_{i j}\right)-\sum_{i \in N_{j}^{-}}\left(x_{j i}+y_{j i}\right)=\left\{\begin{array}{c}
-f, j=0 \\
0, j \neq 0, S, \\
f, j=S
\end{array}\right. \\
0 \leq \mathrm{x}_{\mathrm{ij}} \leq \mathrm{d}_{\mathrm{ij}},
\end{gathered}
$$




$$
0 \leq \mathrm{y}_{\mathrm{ij}} \leq \mathrm{b}_{\mathrm{ij}}
$$

In this situation, there may also be several solutions, that is, several possible maximum flows. Then it is advisable to talk about minimizing the cost of delivering gas to consumers:

$$
\begin{gathered}
\sum_{(i, j)} C_{i j} x_{i j}+\sum_{(i, j)} A_{i j} y_{i j} \rightarrow \min , \\
x \in X^{*} .
\end{gathered}
$$

$f$ - maximal flow, $y_{i j}$-edge's capacity increment (i, $\mathrm{j}) ; A_{i j}$ - price or unit costs for transport of gas by increment $y_{i j} ; b_{i j}$ - edge-increment limits $(\mathrm{i}, \mathrm{j})$.

At the same time, the unit costs for gas transportation by the increment $y_{i j}$ should be many times higher than the unit costs for transporting gas through the grid.

The required bottlenecks are those objects for which, after solving the problem (6) - (11), $\mathrm{y}_{\mathrm{ij}}$ will be greater than zero.

To solve the problem of bypassing the bottlenecks found in the flow distribution model, the possibility of incrementing the gas flow along the edges within $10 \%$ of the capacity of the flow was laid. Such a short-term increase in the capacity of the main gas pipeline section is possible with an increase in the working capacity of compressors at large main compressor stations [9]. As a result of increasing the working pressure in the gas pipeline, an increase in the throughput capacity of the main gas pipeline section is achieved up to $10 \%$. As a result (through the use of the technical capabilities of the gas transportation network), the problem of minimizing gas shortages among consumers is being solved.

\section{Definition of critical combinations of gas industry objects}

The scheme of the Unified Gas Supply System of Russia used for the calculations in this paper on the flow model takes into account all the main features of the operation of the Unified Gas Supply System of Russia and contains:

- 382 nodes, including:

- 28 gas sources;

- 64 gas consumers (subjects of the Russian Federation);

- 24 underground gas storage facilities;

- 266 nodal compressor stations;

- 486 edges representing main gas pipelines and outlets to distribution gas networks.

Initial data, such as daily consumption, extraction, export and import of gas, throughput capacity of existing gas pipelines are adopted in accordance with official statistics [10-12] for 2017. In a specially conducted study [13], an appropriate analysis was carried out, as a result of which 61 objects of the gas industry were assigned to critical gas industry objects. Among these objects there are 25 edges between the node compressor stations and 36 nodes, including 30 nodal compressor stations, 5 head compressor stations at the outlets from large gas fields and a compressor station on one underground gas storage.
Received the above-mentioned 61 objects, calculations were carried out in this settlement scheme to determine critical combinations of objects for each individual critical object. With the subsequent "expansion" of bottlenecks - the implementation of measures aimed at minimizing gas shortage among consumers.

The criterion for assigning a pair to potential critically important combinations was to consider the difference in the $\Delta \mathrm{Q}$ deficit.

$$
\begin{aligned}
& \Delta Q=Q_{\text {pair }}-Q_{o b j}, \\
& \Delta Q \geq \delta .
\end{aligned}
$$

$\mathrm{Q}_{\text {pair }}$ - total gas deficit in the system caused by failure of a couple of objects, $Q_{\text {obj }}$ - gas deficit in case of failure of a critically important object, $\delta$ - restriction of gas deficit increment.

Calculations show that more than 15,000 combinations of other objects with identified critical objects lead to an increase in gas deficit in consumers. Based on considerations of the possibility of expert analysis, we limit the value of $\delta$ to within $5 \%$. As a result of the calculation in pairs of all 61 critical gas industry objects with the rest objects of the system (in total it was analyzed for their simultaneous disconnection of more than 61,000 pairs), 630 pairs were obtained, consisting of one critical object and one gas object, whose failure can lead to the occurrence of $\Delta \mathrm{Q}$ more than $5 \%$.

In table. 1 (the real names of objects of the Unified gas supply system in this article are replaced by conditional serial numbers), the average number of pairs for all objects is clearly represented, as well as the possible total volumes of gas deficit in the system both in the event of critical objects failure, combinations of other gas industry objects with this critically important object. 
Table 1. Critical combinations of objects.

\begin{tabular}{|c|c|c|c|c|c|c|}
\hline \multirow{2}{*}{$\begin{array}{c}\text { Critical } \\
\text { object }\end{array}$} & \multirow{2}{*}{$\begin{array}{l}\text { The number of critical } \\
\text { combinations of objects } \\
\text { (pairs) }\end{array}$} & \multicolumn{2}{|c|}{ Gas deficit, $\%$} & \multirow{2}{*}{$\begin{array}{c}\Delta \mathrm{Q} \\
\%\end{array}$} & \multicolumn{2}{|c|}{ After traversing bottlenecks, $\%$} \\
\hline & & $\begin{array}{l}\text { Single } \\
\text { object }\end{array}$ & Pair & & Gas deficit (pair) & $\begin{array}{c}\text { Decrease of } \\
\text { deficit }\end{array}$ \\
\hline 1,6 & 10 & 21 & $28-32$ & $7-11$ & $25-30$ & $2-3$ \\
\hline $2,5,7$ & 11 & 21 & $28-32$ & $7-11$ & $25-30$ & $2-3$ \\
\hline 3,4 & 8 & 21 & $28-29$ & $7-8$ & $25-26$ & $2-3$ \\
\hline 8 & 7 & 19 & $25-30$ & $6-11$ & $24-29$ & $2-3$ \\
\hline $9-11$ & 5 & 16 & $22-25$ & $6-9$ & $22-25$ & 0 \\
\hline $12-15$ & 7 & 16 & $22-27$ & $6-11$ & $21-25$ & $2-3$ \\
\hline 16 & 5 & 12 & $18-21$ & $6-9$ & $18-19$ & $0-2$ \\
\hline 17 & 14 & 10 & $17-20$ & $7-10$ & $13-18$ & $1-4$ \\
\hline $18-22$ & 5 & 10 & 16-19 & $6-9$ & $13-15$ & $3-4$ \\
\hline 23 & 11 & 10 & $15-17$ & $5-7$ & $11-16$ & $0-4$ \\
\hline 24 & 11 & 9 & $15-17$ & $6-8$ & $11-16$ & $0-4$ \\
\hline 25 & 16 & 8 & $14-20$ & $6-12$ & $12-18$ & $1-4$ \\
\hline 26,27 & 11 & 8 & $13-16$ & $5-8$ & $9-14$ & $0-4$ \\
\hline 28 & 1 & 8 & 14 & 6 & 10 & 4 \\
\hline 29 & 11 & 7 & $13-15$ & $6-8$ & $9-13$ & $0-4$ \\
\hline 32,33 & 11 & 7 & $12-15$ & $5-8$ & $9-13$ & $0-4$ \\
\hline 34,35 & 16 & 7 & $12-19$ & $5-12$ & $10-16$ & $1-4$ \\
\hline $36-39$ & 10 & 7 & 13-18 & $6-11$ & $10-14$ & $3-4$ \\
\hline 40 & 14 & 7 & $13-22$ & $6-11$ & $9-18$ & $3-4$ \\
\hline 41 & 14 & 7 & $13-21$ & $6-14$ & $9-18$ & $3-4$ \\
\hline 42 & 16 & 6 & $12-18$ & $6-12$ & $10-15$ & $1-4$ \\
\hline 43 & 1 & 6 & 12 & 6 & 11 & 1 \\
\hline 45 & 11 & 6 & $11-13$ & $5-7$ & $10-12$ & 1 \\
\hline 46 & 1 & 6 & 11 & 5 & 7 & 4 \\
\hline 47 & 11 & 6 & $11-12$ & $5-6$ & $7-12$ & $0-4$ \\
\hline 48 & 11 & 6 & $11-14$ & $5-8$ & $7-12$ & $0-4$ \\
\hline 49,50 & 16 & 6 & $11-17$ & $5-11$ & $9-15$ & $1-4$ \\
\hline 51 & 24 & 6 & $11-18$ & $5-12$ & $9-15$ & $1-4$ \\
\hline 52 & 9 & 5 & $12-14$ & $7-9$ & $9-10$ & $3-4$ \\
\hline 53 & 19 & 5 & $10-25$ & $5-20$ & $8-22$ & $4-5$ \\
\hline 54 & 17 & 5 & $11-25$ & $6-20$ & $6-22$ & 3-5 \\
\hline 55 & 13 & 5 & $10-25$ & $5-20$ & $6-22$ & $3-5$ \\
\hline 56 & 15 & 5 & $10-22$ & $5-17$ & $6-18$ & $3-5$ \\
\hline 57 & 21 & 5 & $10-19$ & $5-14$ & $6-16$ & $3-5$ \\
\hline 58,59 & 17 & 5 & $10-19$ & $5-14$ & $6-16$ & $3-5$ \\
\hline 60,61 & 20 & 5 & 10-19 & $5-14$ & $6-16$ & $3-5$ \\
\hline
\end{tabular}

Table. 1 show that apart from some critically important objects $(28,43,46)$ having only one critically important combination of objects (one pair each), from 5 to 24 critically important combinations of objects were obtained for the remaining critical objects. So the first seven critical objects are present in 8 to 11 critical combinations of objects, the failure of which will entail a total daily gas deficit for consumers in the system as a whole in the amount of $27-32 \%$. The difference in the deficit $\Delta \mathrm{Q}$ for these pairs is $7-11 \%$.

In general, the following should be noted from the results of the study. Violation of the functioning of a critical combination of objects can lead to a greater gas deficit in consumers by an average of $8-10 \%$ than in the case of a violation of the functioning of a particular critical object.

In possible situations with the failure of the critical combinations of objects considered, measures to bypass bottlenecks result in an insignificant decrease in the gas deficit (on average by $2-3 \%$ ), which also confirms the high importance of identifying these combinations. Here it is worth noting the critical combination of objects with critical objects 9-11, to which measures to bypass bottlenecks have no effect, that is, that before, that after the bypassing of bottlenecks the same volumes of gas deficit remain.

It is necessary to highlight 4 objects - 2 nodal compressor stations and 2 edges between the nodal compressors stations, which are present in $60 \%$ of the critical combinations of objects obtained. Violation of the normal functioning of these facilities together with a number of critical objects can significantly increase the gas deficit in consumers. 


\section{Conclusion}

As a result of this research, the issues of searching for critical combinations of gas industry objects were considered. The definition of such objects in the gas industry, failure of which, coupled with one of the critical objects, will lead to a much larger gas deficit in consumers than in the event of failure of these objects separately. To search for critical combinations of gas industry objects, the flow distribution model laid in the software "Oil and Gas of Russia" was used. This model is designed to assess the production capabilities of the gas transmission network in conditions of various kinds of disturbances. A detailed analysis of the major critical combinations of gas industry objects is given.

The conducted studies showed that in the modern configuration of the Unified Gas Supply System, situations are possible when, in the event of a critical combination of objects failing, the total gas deficit in consumers can reach $32 \%$ of the total gas demand. Research and accounting of such objects and their combinations is necessary to improve the reliability of the Unified Gas Supply System in its development and reconstruction works. The main efforts in this case should be aimed at reducing the conditional significance of the identified specific critical combinations of objects for the operability of the entire system.

The work was carried out within the framework of a scientific project III.17.5.1 of program of fundamental research of the SB RAS, reg. number AAAA-A17-117030310451-0.

\section{References}

1. N.I. Pyatkova, [and others], Energy security of Russia: problems and solutions, 198 p. (2011).

2. S.M. Senderov, V.I. Rabchuk, A.V. Edelev, Izv. Ros. Akad. Nauk. Energetika. № 1. p. 70-78. (2016).

3. S. Senderov, A. Edelev, Energy. doi: $10.1016 /$ J.ENERGY.2017.11.063. (2017).

4. S. Vorobev, A. Edelev, MLSD 2017, IEEE, 2017. DOI 10.1109 / MLSD.2017.8109707. (2017).

5. S. Vorobev, A. Edelev, E. Smirnova, RSES 2017. E3S Web Conf. Vol. 25, DOI 10.1051 / e3sconf / 20172501004. (2017).

6. A.V. Edelev, S.M. Enikeeva, S.M. Senderov, Computational technologies. - Vol. 4, № 5. - p. 30 - 35 . (1999).

7. S.V. Vorobev, A.V. Edelev, Software products and systems. - №3. - p. 174 - 177. (2014).

8. L.R. Ford, D.R. Fulkerson. Flows in networks. Princeton University Press. 276p. (1966).

9. Yu.P. Korotaeva, R.D. Margulova, Extraction, preparation and transport of natural gas and condensate. Reference manual in 2 volumes. Volume II. Nedra, 288 p., (1984).

10. Export of the Russian Federation of the most important goods in 2011 - 2016 (according to the Federal Customs Service of Russia) http://customs.ru/ index.php?option=com _ newsfts\&view =category\&id= 52\&Itemid $=1978 \&$ limitstart $=60$.

11. InfoTEK Monthly oil and gas magazine.

№1, - P.154. (2017).

12. Ministry of Energy of the Russian Federation. Statistics. http://minenergo.gov.ru/activity/statistic.J. Johansson, H. Hassel, E. Zio, Reliability Engineering \& System Safety. No. 120. P.27-38. (2013).

13. S.M. Senderov, V.I. Rabchuk, S.V. Vorobev, Proc. of the col. of rep. Methodological issues of reliability research of large energy systems. 90th meeting "Reliability of developing energy systems". July 1-7, Irkutsk. (2018). 